\title{
Inelastic neutron scattering due to acoustic vibrations confined in nanoparticles: theory and experiment
}

\author{
Lucien Saviot, ${ }^{1}$ Caleb H. Netting, ${ }^{2}$ Daniel B. Murray, ${ }^{2}$ Stéphane Rols, ${ }^{3}$ Alain \\ Mermet, ${ }^{4}$ Anne-Laure Papa, ${ }^{1}$ Catherine Pighini, ${ }^{1}$ Daniel Aymes, ${ }^{1}$ and Nadine Millot ${ }^{1}$ \\ ${ }^{1}$ Institut Carnot de Bourgogne, UMR 5209 CNRS - Université de Bourgogne, \\ 9 avenue A. Savary, BP 47870, F-21078 Dijon Cedex, France* \\ ${ }^{2}$ Mathematics, Statistics and Physics Unit, \\ The University of British Columbia Okanagan, 3333 University Way, \\ Kelowna, British Columbia, Canada V1V 1 V州 \\ ${ }^{3}$ Institut Laue Langevin, 6 rue Jules Horowitz, BP 156, 38042 Grenoble, France \\ ${ }^{4}$ Université de Lyon, F-69622, Lyon, France; \\ Université Lyon 1, Villeurbanne; \\ CNRS, UMR5620, Laboratoire de Physico-Chimie des Matériaux Luminescents
}

(Dated: September 13, 2018)

\begin{abstract}
The inelastic scattering of neutrons by nanoparticles due to acoustic vibrational modes (energy below $10 \mathrm{meV}$ ) confined in nanoparticles is calculated using the Zemach-Glauber formalism. Such vibrational modes are commonly observed by light scattering techniques (Brillouin or low-frequency Raman scattering). We also report high resolution inelastic neutron scattering measurements for anatase $\mathrm{TiO}_{2}$ nanoparticles in a loose powder. Factors enabling the observation of such vibrations are discussed. These include a narrow nanoparticle size distribution which minimizes inhomogeneous broadening of the spectrum and the presence of hydrogen atoms oscillating with the nanoparticle surfaces which enhances the number of scattered neutrons.
\end{abstract}




\section{INTRODUCTION}

Due to the growing interest in nanoscale materials during the last decades, the confined acoustic vibrations of nanoparticles (NP) have been investigated both theoretically and experimentally. Their frequency is roughly on the order of $v / d$ where $v$ is the speed of sound and $d$ is the diameter and is on the order of $1 \mathrm{THz}$ for a typical NP.

Low-frequency Raman scattering experiments can observe such vibrations. This can be done either for free nanoscale objects or for NPs embedded in a matrix with large impedance mismatch. $\stackrel{1}{*}$ Numerous Raman observations of these vibration modes are available in the literature (see references citing 2). These same vibrations can also be observed using time resolved pump-probe experiments ${ }^{3}$ and far-infrared absorption..$^{4,5}$

In this work, we investigate the possibility of observing confined acoustic vibrations through inelastic neutron scattering (INS). While neutrons interact with the nuclei of the atoms and therefore directly probe their motion, incident photons involved in the Raman scattering, far-infrared absorption and pump-probe experiment processes interact with electrons. The vibrations of the atoms are thus probed through the electron-vibration interaction. A distinct feature of INS is the absence of selection rules allowing the complete vibration bands to be measured in one experimental set. In particular, the so-called generalized density of states (GDOS) can be measured in polycrystalline powders. In the low frequency range, for usual bulk 3D crystals the Debye regime is observed with a GDOS having a $\omega^{2}$ dependence. There are some past INS studies on nanoscale systems which indicated a generally elevated background level which could be attributed to INS from acoustic vibrations. $.7,8,9,10,11,12$ However, what has been missing up until now has been the association of a distinct spectral feature. A key requirement for accomplishing this is that the sample has a sufficiently uniform collection of NPs so that the frequencies of interest have a narrow distribution. This enables the observation of the size dependence of the spectral features. 


\section{EXPERIMENT}

\section{A. Samples}

Unlike previous INS experiments where observations of confined acoustic phonons were attempted, we started from a set of samples for which confined acoustic modes have already been observed. $\mathrm{TiO}_{2}$ nanopowders have been prepared by continuous hydrothermal synthesis as detailed elsewhere $\stackrel{\underline{13}}{\underline{3}}$ Some of us previously reported low-frequency Raman scattering $\underline{13}$ of these samples. Even some far infra-red features ${ }^{4}$ have been tentatively attributed to confined acoustic vibrations. The observed low-frequency Raman peaks are narrower than the ones previously observed in the literature ${ }^{14,15,16}$ for anatase $\mathrm{TiO}_{2}$ nanopowders which is in agreement with the narrow size distribution determined by transmission electron microscopy (TEM). The sizes determined by the broadening of the X-ray diffraction (XRD) patterns are also in good agreement with those determined by TEM indicating that the NPs are mostly monocrystalline as XRD measurements are sensitive to the size of the coherent domains. Two different powders have been probed by INS to verify size dependence. The first one, referred to as HT-5 as in Ref. 13, contains NPs with an average diameter of $3.6 \mathrm{~nm}$ while the second sample HT-7 has larger NPs whose average diameter is $6.5 \mathrm{~nm}$. The full width at half maximum (FWHM) of the size distribution is close to $60 \%$ of the average size for both samples. These values were obtained by analyzing the low-frequency Raman peak as in a previous work ${ }^{13}$ but using the Resonant UltraSound (RUS) model ${ }^{17}$ instead of Lamb's model. 18 The agreement with the average size obtained by XRD and BET measurements but also with the size distribution obtained from HRTEM photos 19 is improved.

As checked by thermogravimetry measurements, a significant amount of species are adsorbed at the surface of the nanopowders ${ }^{20,21}$ For our samples, typically $10 \%$ of the total mass could be removed from the sample by applying a low vacuum. Further temperature annealing helps to remove more but it also can change the NP size and their stoechiometry for temperatures above $500 \mathrm{~K} .^{20}$ It is well-known that anatase $\mathrm{TiO}_{2}$ surfaces are stabilized by adsorption of water molecules and formation of $\mathrm{OH}$ bonds. ${ }^{22,23}$ Indeed, X-ray Photoemission

Spectroscopy (XPS) measurements (which require ultra-high vacuum) show that for sample HT-7, more than $10 \%$ of the total number of atoms are hydrogen atoms. ${ }^{19}$ The neutron scattering cross section of a single hydrogen atom is roughly 20 times more than that of a 
single oxygen or titanium atom. Therefore, in this ultra-high vacuum condition the contribution of hydrogen atoms to the total scattering would be more than $\frac{2}{3}$. This value is the worst case estimate of the minimum contribution expected in this work as the proportion of hydrogen atoms increases when decreasing the size but also when more water molecules are adsorbed as is the case during INS measurements because the vacuum is not as good.

Due to the high scattering cross-section of hydrogen compared to oxygen and titanium, it is not clear if neutron scattering from hydrogen could hinder the observation of the NPs vibrational density of states as in a recent work ${ }^{24}$ or instead enhance it if hydrogen atoms move with the NPs' surface. Therefore some heat treatments were performed to check the influence of adsorbed water.

\section{B. Measurements}

INS experiments have been performed with the IN6 time-focusing time of flight spectrometer at the Institut Laue-Langevin (ILL). For the first experiments, the neutron wavelength was $5.1 \AA$. For each sample, $500 \mathrm{mg}$ of powder were wrapped inside a non-hermetic aluminum foil and put inside a cryofurnace. The empty foil signal was removed and the efficiencies of the detector were corrected using a vanadium sample run. Measurements were carried out first at $300 \mathrm{~K}$, then the samples were heated at $500 \mathrm{~K}$ and annealed for a few hours. During annealing, the helium atmosphere surrounding the sample was purged a few times to removed desorbed water. Then further measurements were performed at 300, 200, 100 and $10 \mathrm{~K}$ to check for a quasielastic signal.

Another round of experiments was performed later in order to have better statistics. The neutron wavelength was $4.1 \AA$, the temperature was $500 \mathrm{~K}$ and the accumulation time much longer. The aluminium foil was inside a cryoloop. In this configuration, the foil was more hermetic allowing to have more water molecules adsorbed at the surface of the NPs.

\section{Results}

The incoherent neutron scattering functions $S(\omega)$ measured on sample HT5 at 300K be-

fore and after heat treatment at $500 \mathrm{~K}$ are presented in Fig. 1, It was obtained by summing $S(\theta, \omega)$ over all available detector angles after checking that it doesn't depend on the scat- 


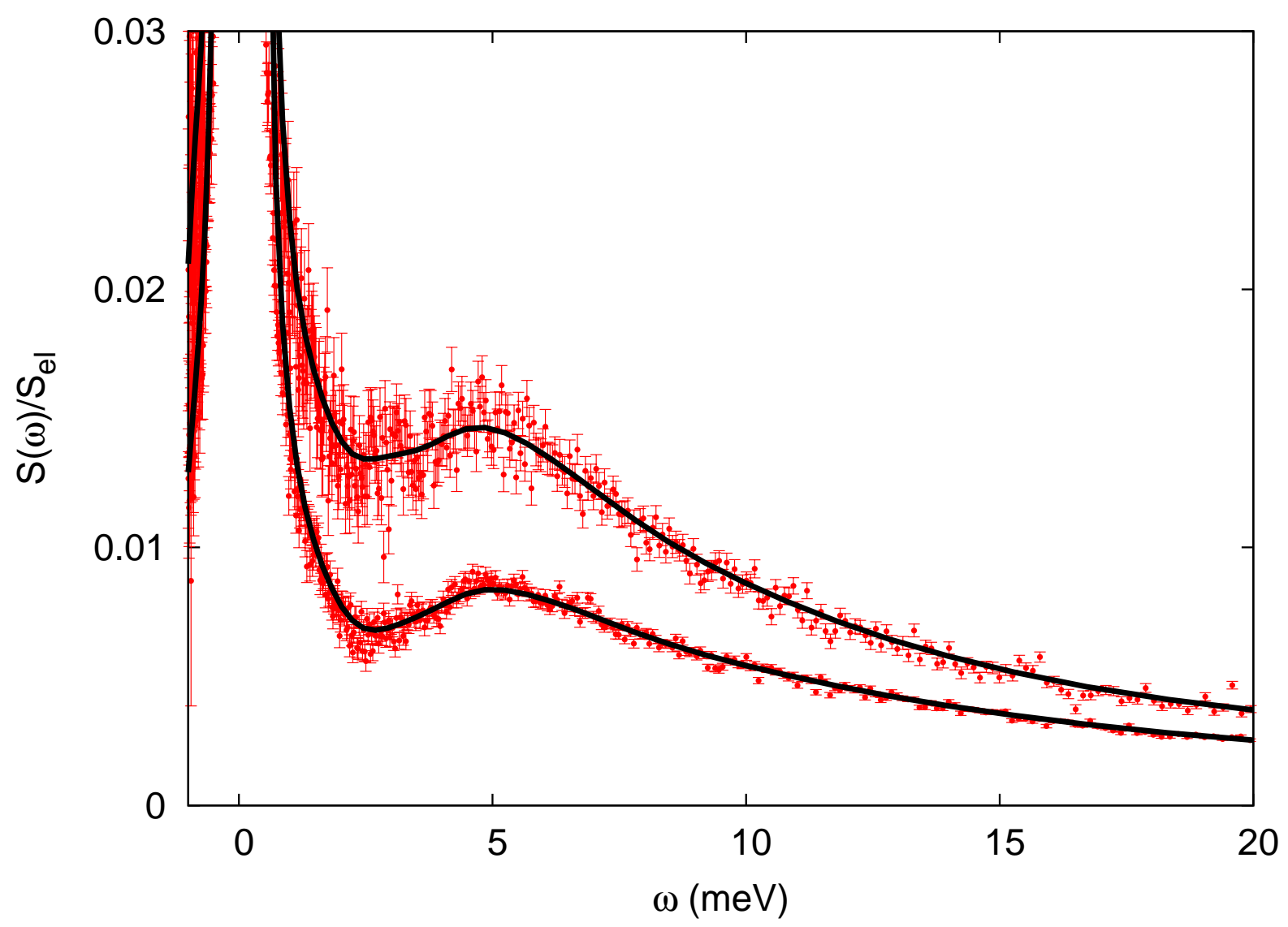

FIG. 1: (Color online) $S(\omega) / S_{e l}$ with experimental error bars for an anatase $\mathrm{TiO}_{2}$ nanopowder with nanoparticles having an average diameter $d=3.6 \mathrm{~nm}$ (sample HT5). The neutron wavelength is $5.1 \AA$ and the temperature is $\mathrm{T}=300 \mathrm{~K}$ before (top) and after (bottom) the heat treatment and atmosphere replacement at 500K. The black lines obtained by smoothing the data are only a guide for the eye.

tering wavevector. These spectra have been normalized by the area under the elastic peak $\left(S_{e l}\right)$ obtained by fitting by a Gaussian. Error bars are also drawn representing the experimental error. This error is obtained by propagating through all the calculations the error (square root of the number of neutrons) for each detector and time channel. The significant decrease of the inelastic intensity is clearly related to the desorption of water which occurred during the heat treatment and also because the atmosphere was purged during this process. As a result, there are fewer hydrogen atoms at the surface of the $\mathrm{TiO}_{2} \mathrm{NPs}_{\mathrm{s}}$ after the heat treatment. A peak is clearly identified close to $5 \mathrm{meV}$ and its position is the same before and after the heat treatment. 


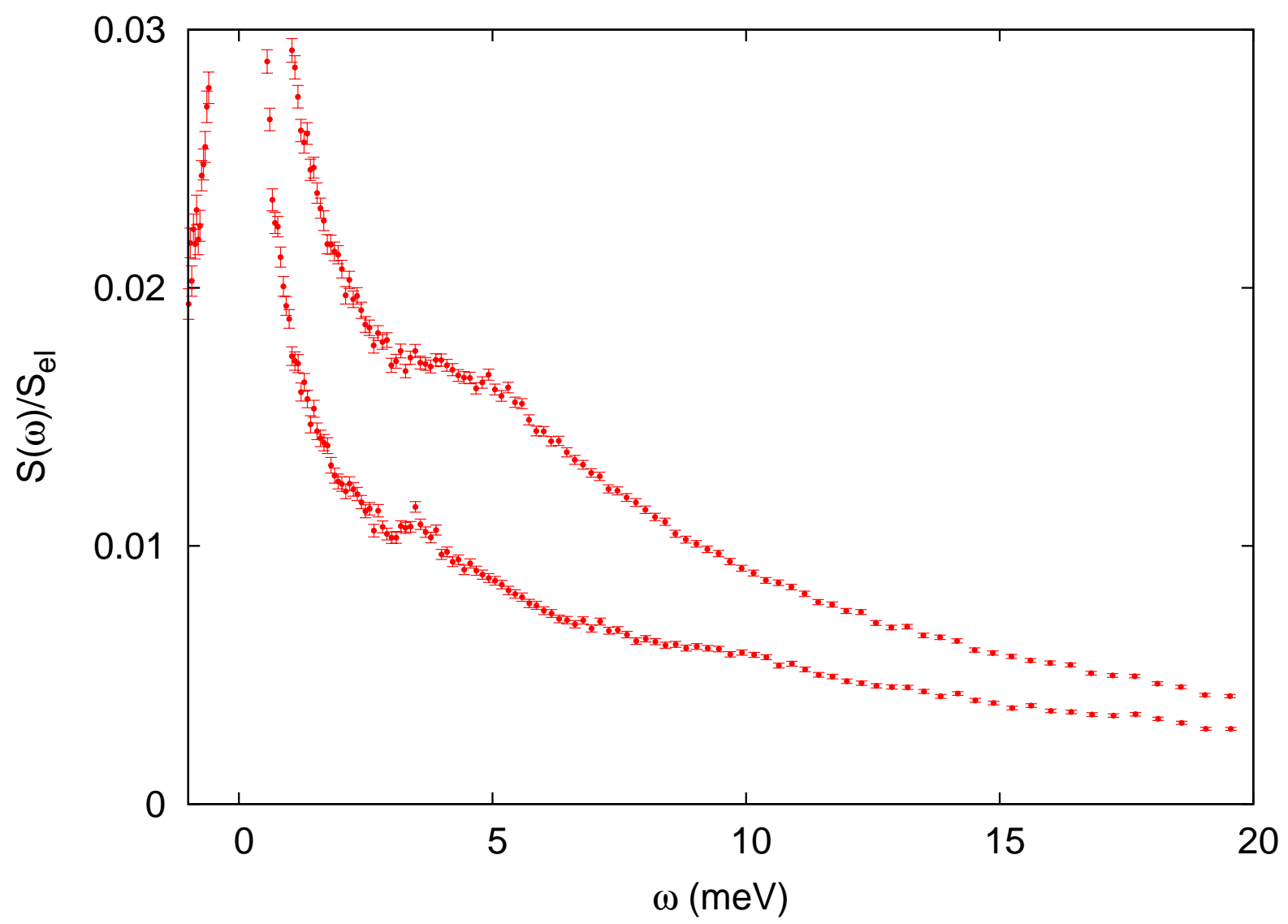

FIG. 2: (Color online) $S(\omega) / S_{e l}$ with experimental error bars for two anatase $\mathrm{TiO}_{2}$ nanopowders with nanoparticles having an average diameter $d=3.6 \mathrm{~nm}$ (sample HT5, top) and $d=6.5 \mathrm{~nm}$ (sample HT7, bottom). The neutron wavelength is $4.1 \AA$ and the temperature $\mathrm{T}=500 \mathrm{~K}$.

Similar experiments were performed for sample HT7 but the signal measured was smaller preventing the observation of a similar low-frequency peak. This signal could be improved by increasing the temperature and keeping more hydrogen at the surface in the second round of experiments at the expense of an increased quasielastic signal. Figure 2 presents the measured $S(\omega)$ for samples HT5 and HT7 at 500K. The shape of the spectrum clearly depends on the size of the nanoparticles as the peak observed for sample HT5 is not present for sample HT7. This change is compatible with a shift of the peak towards lower energies as the radius of the nanoparticle increases. 


\section{THEORY}

\section{A. Inelastic neutron scattering}

There is no theoretical formalism presently available which is developed specifically for INS from a NP powder sample. Since the nanopowder used in this work is of low density, it is a reasonable approximation to treat the constituent NPs as mechanically independent, each NP vibrating as if free. Thus, the INS due to a single free NP can be theoretically predicted and the result applied to the entire sample, taking into consideration the size distribution of the nanopowder. The total neutron scattering cross section is simply additive. Under our free NP approximation, there is no coherence between the mechanical vibrations of different NPs.

Notations are the same as in Ref. 25. Consider a single NP consisting of atoms which are labeled by integer index $\nu$. Atom $\nu$ has mass $m_{\nu}$ and scattering length $a_{\nu}$. As explained in Ref. 25, $a^{2}=1 /(4 \pi)\left(\sigma_{c o h}+\sigma_{\text {inc }}\right)$ where $\sigma_{c o h}$ and $\sigma_{\text {inc }}$ are, respectively, the coherent and incoherent bound cross sections for the given type of atom. The NP has vibrational modes indexed by $\lambda$, with frequencies $\omega_{\lambda}$ and mass-weighted mode displacements $\vec{e}_{\nu}^{(\lambda)}$. A general vibrational state transition is indicated by a set of integers $\left[n_{\lambda}\right]$ so that the energy gain of the neutron after the scattering is $\hbar \omega$ where

$$
\omega=-\sum_{\lambda} n_{\lambda} \omega_{\lambda}
$$

The initial wavevector of the neutron is $\vec{k}_{o}$ with magnitude $k_{o}$. The final wavevector is $\vec{k}$ with magnitude $k$. The change of wavevector is $\vec{k}=\vec{k}-\overrightarrow{k_{o}}$.

Using previous theories on the differential cross section of INS due to vibrational modes of a molecule $e^{25}, 26$, a formula for the neutron scattering cross section due to a given general vibrational state transition of a molecule can be written as:

$$
<\sigma\left(\left[n_{\lambda}\right], \theta\right)>_{T}=\frac{k}{k_{o}} \sum_{\nu} a_{\nu}^{2} W_{\nu}
$$

where

$$
\begin{aligned}
W_{\nu}= & \prod_{\lambda} \exp \left[-\left(\frac{\hbar}{2 m_{\nu} \omega_{\lambda}}\right)\left(\vec{\kappa} \cdot \vec{e}_{\nu}^{(\lambda)}\right)^{2} \operatorname{coth}\left(\frac{\hbar \omega_{\lambda}}{2 k_{B} T}\right)\right] \\
& \times \exp \left(\frac{-n_{\lambda} \hbar \omega_{\lambda}}{2 k_{B} T}\right)
\end{aligned}
$$




$$
\times I_{n_{\lambda}}\left(\frac{\left(\hbar / 2 m_{\nu} \omega_{\lambda}\right)\left(\vec{\kappa} \cdot \vec{e}_{\nu}^{(\lambda)}\right)^{2}}{\sinh \left(\hbar \omega_{\lambda} / 2 k_{B} T\right)}\right)
$$

and $I_{n_{\lambda}}$ is a modified Bessel function of order $n_{\lambda}$.

This expression ignores off-diagonal contributions to $\sigma$ which come from coherent interference between different atoms. The formula is valid whether the neutron scattering is coherent or incoherent. As in Hudson et al $\stackrel{25}{ }$, the contribution to the total cross section from the off-diagonal terms is expected to be negligible. In the following, the coherent scattering terms are omitted because as discussed before most of the experimental scattering events are due to the incoherent scattering by hydrogen atoms. However, we expect the motion of these atoms to be representative of the motion of the surface of the NP. This is true for the $\mathrm{OH}$ bonds at the surface because the acoustic phonon frequencies are very small compared to the frequency of the $\mathrm{OH}$ bond. The situation for water molecules physisorbed on the surface is of course more complex. However the atmosphere was purged, the helium atmosphere pressure was around 10 mbar and the temperature was increased in order to reduce the amount of such molecules. As a result, it is safe to neglect the contribution of these molecules.

In contrast to many INS observations of vibrational modes of molecules, the above expressions can be substantially simplified for the experimental situation we are considering because of the large mass of the NP and also the long wavelength of the incident neutrons (which makes $k_{o}$ small). For both the first and third factors in Eq. (3), the function argument is on the order of $k^{2} k_{B} T / \omega^{2} M$, where $M$ is the mass of a NP. At room temperature, this is approximately 0.00002 , so linear approximations of the functions can thus be used and higher corrections are thus negligible. The first factor is the Debye-Waller factor. It is well-approximated as 1 in every situation relevant to our experiment. This is partly a result of the smallness of $\kappa$ for this experimental setup. It is also related to the low frequency involved. For the third factor, we make use of the limiting form of the modified Bessel function $I_{n}(x) \simeq(x / 2)^{|n|} /(|n| !)$. Since $x$ is so small, only terms with $n=-1$ or +1 need to be included. In our experiment, we only collect data for increasing neutron energy, which corresponds to $n_{\lambda}=1$. We are now only talking about a single mode with frequency $\omega$, so we no longer mention $\lambda$. Then,

$$
W_{\nu}=\frac{\hbar}{4 m_{\nu} \omega} \exp \left(\frac{-\hbar \omega}{2 k_{B} T}\right) \frac{\left(\vec{\kappa} \cdot \vec{e}_{\nu}\right)^{2}}{\sinh \frac{\hbar \omega}{2 k_{B} T}}
$$


The next step is to consider the random orientation of the NPs. As a result, the orientation of the motion of a given atom is random with respect to the direction of $\vec{\kappa}$. Thus, $\left(\vec{\kappa} \cdot \vec{e}_{\nu}\right)^{2}$ can be replaced by $\frac{1}{3} \kappa^{2} e_{\nu}^{2}$.

To evaluate the total inelastic cross section for this mode (to be called $\sigma_{\text {inel }}$ ) we have to integrate the differential cross section $\sigma(\theta)$ over all possible final directions of the final neutron wavevector $\vec{k}$. $\theta$ being the angle between $\vec{k}$ and $\overrightarrow{k_{o}}$,

$$
\kappa^{2}=k^{2}+k_{o}^{2}-2 k k_{o} \cos \theta
$$

Therefore

$$
<\kappa^{2}>=k^{2}+k_{o}^{2}-2 k k_{o}<\cos \theta>
$$

This expression depends on $\omega$ as $k_{o}$ is fixed by the experimental conditions, $k=$ $\sqrt{k_{o}^{2}+\frac{2 m_{n} \omega}{\hbar}}$ and $\langle\cos \theta>$ can be calculated from the geometry of the experimental setup. Here, we took $<\cos \theta>=0.2804$.

The final result is a nearly exact expression for the inelastic cross section:

$$
\begin{array}{r}
\sigma_{\text {inel }}=k^{2}\left(\frac{k}{k_{o}}+\frac{k_{o}}{k}-2<\cos \theta>\right) \\
\times \exp \left(\frac{-\hbar \omega}{2 k_{B} T}\right) \frac{1}{12} \frac{\hbar}{\omega} \frac{1}{\sinh \left(\hbar \omega / 2 k_{B} T\right)} \\
\times \sum_{\nu}\left(\frac{\sigma_{\nu} e_{\nu}^{2}}{m_{\nu}}\right)
\end{array}
$$

\section{B. Acoustic modes and size distribution}

Let us first figure out the summation over the different atoms $(\nu)$ of $\frac{\sigma_{\nu} e_{\nu}^{2}}{m_{\nu}}$. Summations over all modes as well as the size distribution of NPs in order to get a quantity closer to the experimentally INS spectrum will be considered later. Let $\sigma_{H}$ and $m_{H}$ respectively denote the neutron scattering cross section and mass of a hydrogen atom. Assuming that only the scattering by $\mathrm{H}$ surface atoms matters and neglecting the scattering by other atoms ( $\mathrm{Ti}$ and $\mathrm{O}$ ), we can rewrite this summation as being over all the $\mathrm{H}$ atoms $\frac{\sigma_{H}}{m_{H}} \sum_{\nu_{H}} e_{\nu_{H}}^{2} \cdot \rho$ is the density of the NP and $V$ is the volume. $N_{H}$ is the number of hydrogen atoms fixed to the surface of the NP. Introducing the mean square displacement at the surface U2S defined as the ratio of the surface and volume average of the square displacement as in Ref. 27, it 
follows that:

$$
\begin{aligned}
\sum_{\nu}\left(\frac{\sigma_{\nu} e_{\nu}^{2}}{m_{\nu}}\right) & =\frac{\sigma_{H}}{\rho} \frac{N_{H}}{V} \mathrm{U} 2 \mathrm{~S} \\
& =\frac{\sigma_{H} d_{H}}{\rho} \frac{3}{R} \mathrm{U} 2 \mathrm{~S}
\end{aligned}
$$

where $d_{H}$ is the surface density of hydrogen atoms which we will suppose to be independent of $R$ in the following. The summation above is over the hydrogen atoms only. This expression already shows that vibrational modes having a large amplitude at the surface and larger NPs will scatter neutrons more.

As with most low-frequency Raman interpretations, we model the nanopowder as an ensemble of spherical elastic NPs. The spherical shape is consistent with the average shape observed by TEM. All the frequencies of homogeneous elastically anisotropic spheres $\omega_{\lambda}(R)=\frac{\alpha_{\lambda}}{R}$ can be calculated using the method introduced by Visscher et al. 17 Taking into account the degeneracy of the different modes and the radius distribution $P(R)$, the incoherent neutron scattering function can be calculated. For an ideal system consisting of an ensemble of identical NPs, it would be non-zero only for the discrete set of frequencies of the NPs. For a real system, these discrete frequencies are broadened and the maxima are shifted by the distribution of NPs.

For a single vibration mode and a single particle, we obtain the following expression for incoherent INS due to a uniform distribution of $\mathrm{H}$ atoms on the surface of the nanosphere:

$$
\sigma_{\text {inel }}\left(\omega_{\lambda}\right) \sim \frac{1}{\alpha_{\lambda}} \frac{\kappa^{2}}{k_{o} k} \frac{\mathrm{U}_{2} \mathrm{~S}_{\lambda}}{\exp \left(\frac{\hbar \omega_{\lambda}}{k_{B} T}\right)-1}
$$

where only the terms depending on $R$ or $\lambda$ have been retained. It should be noted that $\mathrm{U}_{2} \mathrm{~S}_{\lambda}$ does not depend on $R$.

Now summing over the different modes $\lambda$ and radii $R$ we obtain:

$$
S(\omega) \sim \sum_{\lambda} \mathrm{U}_{2} \mathrm{~S}_{\lambda} \int_{R} \frac{\kappa^{2}}{k_{o} k} \frac{P(R) \delta\left(\omega-\omega_{\lambda}(R)\right) d R}{\alpha_{\lambda}\left(\exp \left(\frac{\hbar \omega}{k_{B} T}\right)-1\right)}
$$

Using the properties of the $\delta$ function, this expression translates into:

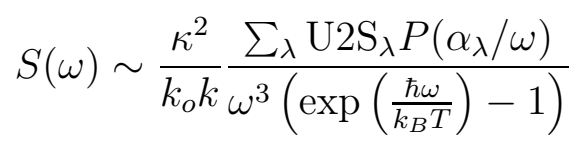

By using the RUS algorithm $\frac{17}{}$ and the anisotropic elastic parameters for anatase $\mathrm{TiO}_{2}$ from Ref. 28 we could calculate the $\omega_{\lambda}$ for nanospheres, the associated displacements and 
their classification according to their irreducible representation. This approach relies on continuum elasticity. The shape and elastic tensor for a given object are defined. Then the

displacements of the eigenmodes are expanded on a $x^{i} y^{j} z^{k}$ basis. For the free boundary condition, the eigensolution problem is turned into a real generalized symmetric-definite eigenproblem through the use of the Hamilton's principle. Such a problem is efficiently solved on any modern computer. Using this method, we could calculate the $\mathrm{U}_{2} \mathrm{~S}_{\lambda}$ by numerical integration. Some values for the first few modes are presented in Tab. \. Calculations for sample HT5 could then be performed and are presented in Fig. 3 using the average size for sample HT-5 and different Gaussian size distributions. For comparison with the frequently used isotropic model, the anisotropic eigenmodes obtained with the RUS algorithm have been projected onto the Lamb's modes and the main contributions are shown in the last column of Tab. I. This demonstrates the splitting and mixing of the isotropic modes due to the anisotropy of the tetragonal elasticity similarly to what has been recently reported for the cubic symmetry. ${ }^{29}$

\section{DISCUSSION}

\section{A. General agreement and considerations regarding the vibrations}

As shown by Fig. 3, the discreteness of the acoustic vibrations in NPs is expected to be observed in the INS spectrum in a similar way that such vibrations can be observed by inelastic light scattering experiments. Of course, the size distribution plays a significant role and may hide the narrow features expected for a single NP. This effect is more dramatic for INS because of the absence of selection rules which makes all vibration modes observable.

Apart from the elastic and quasielastic contributions which are not taken into account in our calculations, the calculated $S(\omega)$ in Fig. 3 is qualitatively in agreement with the experimental spectra for sample HT-5 in Fig. 1 and 2. The main feature is a peak around $3 \mathrm{meV}$ resulting mainly from the lowest frequency vibrations and the frequency gap in the long wavelength regime, extending from 0 up to about $2 \mathrm{meV}$. The position of the experimental peak for this sample is close to $5 \mathrm{meV}$ to be compared with the calculated position at $3 \mathrm{meV}$. This discrepancy will be discussed in the following section. The other features at higher frequencies are progressively smoothed when increasing the width of the 


\begin{tabular}{|c|c|c|c|}
\hline$\omega(\mathrm{meV})$ & $\mathrm{U} 2 \mathrm{~S}$ & $\mathrm{IR}$ & projection onto Lamb's modes \\
\hline 3.26 & 0.9 & $\mathrm{~A}_{1 g}$ & $(\mathrm{SPH}, \ell=2)$ \\
3.26 & 1.9 & $\mathrm{E}_{u}$ & $(\mathrm{TOR}, \ell=2)$ \\
3.43 & 1.9 & $\mathrm{~A}_{1 u}$ & $(\mathrm{TOR}, \ell=2)$ \\
3.49 & 1.8 & $\mathrm{~B}_{1 u}$ & $(\mathrm{TOR}, \ell=2)$ \\
3.63 & 0.9 & $\mathrm{E}_{g}$ & $(\mathrm{SPH}, \ell=2)$ \\
3.76 & 0.9 & $\mathrm{~B}_{2 g}$ & $(\mathrm{SPH}, \ell=2)$ \\
3.80 & 1.7 & $\mathrm{~B}_{2 u}$ & $(\mathrm{TOR}, \ell=2)$ \\
4.38 & 0.9 & $\mathrm{~B}_{1 g}$ & $(\mathrm{SPH}, \ell=2)$ \\
4.97 & 1.0 & $\mathrm{~A}_{2 u}$ & $(\mathrm{SPH}, \ell=1)+(\mathrm{SPH}, \ell=3)$ \\
5.09 & 2.0 & $\mathrm{~B}_{2 g}$ & $(\mathrm{TOR}, \ell=3)$ \\
5.12 & 1.2 & $\mathrm{E}_{u}$ & $(\mathrm{SPH}, \ell=1)+(\mathrm{SPH}, \ell=3)$ \\
5.14 & 1.1 & $\mathrm{~A}_{2 u}$ & $(\mathrm{SPH}, \ell=3)+(\mathrm{SPH}, \ell=1)$ \\
5.23 & 2.0 & $\mathrm{E}_{g}$ & $(\mathrm{TOR}, \ell=3)$ \\
5.32 & 1.0 & $\mathrm{E}_{u}$ & $(\mathrm{SPH}, \ell=3)$ \\
5.41 & 2.1 & $\mathrm{~A}_{2 g}$ & $(\mathrm{TOR}, \ell=3)$ \\
$\vdots$ & $\vdots$ & $\vdots$ & $\vdots$ \\
\hline
\end{tabular}

TABLE I: Frequencies, U2S and irreducible representations (IR) for the vibration modes of a $d=3.6 \mathrm{~nm}$ anatase $\mathrm{TiO}_{2}$ NP. Degeneracy is 1 except for E modes for which it is 2 . The last column show the correspondence between the spheroidal (SPH) and torsional (TOR) Lamb's modes having an angular momentum $\ell$ of an isotropic sphere.

size distribution because the frequency gaps are too narrow.

Instead of using the numerical RUS approach to calculate the NP vibration modes and frequencies, it would have been simpler to use the isotropic approximation (Lamb's model ${ }^{18}$ ) for which an exact solution exists. However, using such a model would have resulted in artificially narrow peaks in the INS spectrum because the vibration mode degeneracy is higher in that case. A single domain spherical anatase $\mathrm{TiO}_{2}$ nanocrystal has tetragonal symmetry and the isotropic vibrations are split into modes having degeneracy at most two for this symmetry. Therefore not using the isotropic approximation enables us to have more accurate calculations of the frequencies and U2S but also prevents the appearance of some 


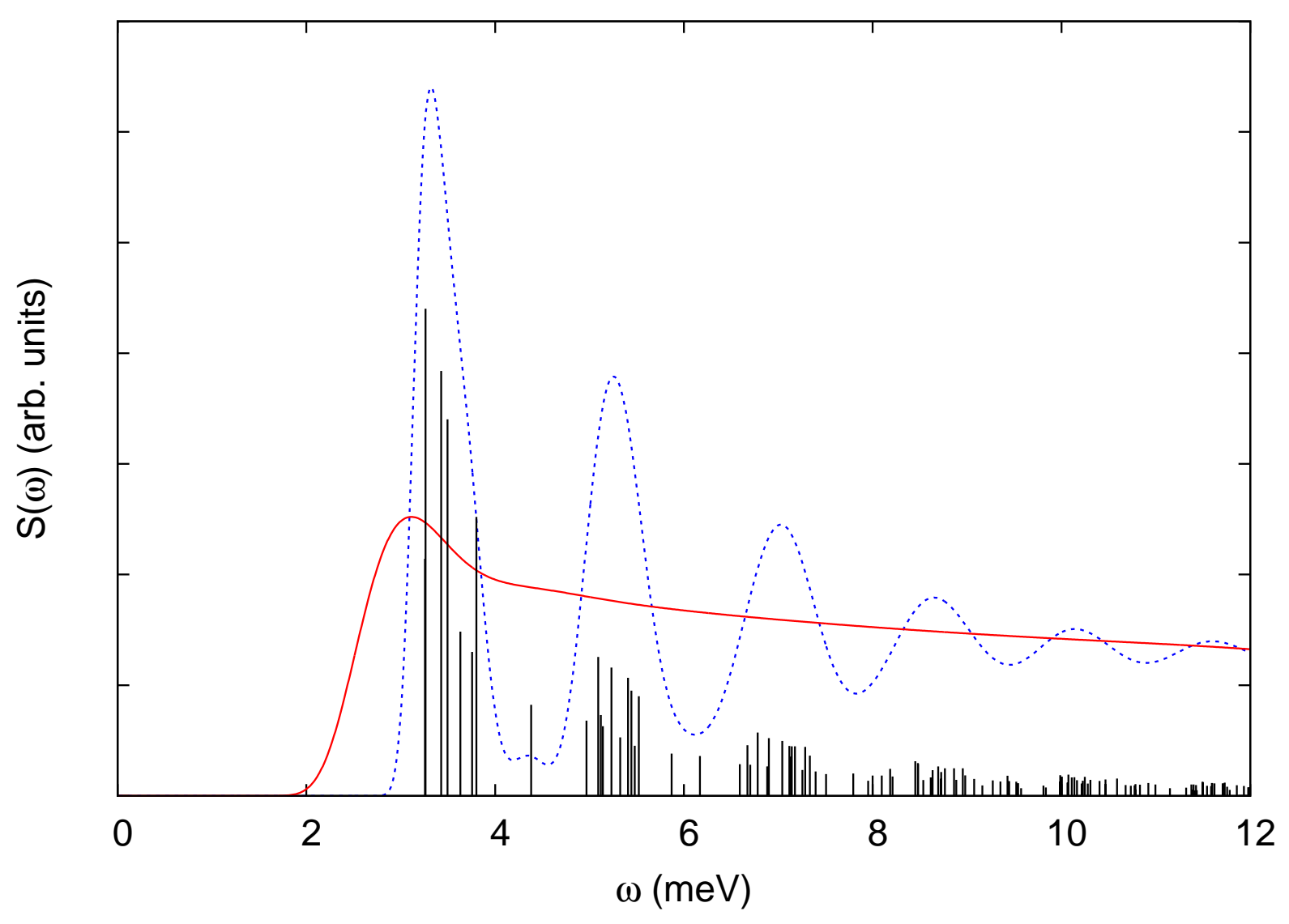

FIG. 3: (Color online) $S(\omega)$ calculated for sample HT5 with varying Gaussian size distribution. The average diameter is $d=3.6 \mathrm{~nm}$ in all cases. The FWHM of the size distribution is $10 \%$ and $50 \%$ of the average size for the dotted and continuous curves respectively. The neutron wavelength is $5.1 \AA$. The vertical lines at the bottom show the theoretical frequencies of the eigenvibrations for a spherical particle having a $3.6 \mathrm{~nm}$ diameter. The height of these lines is proportional to the degeneracy of the modes (either 1 or 2 ) multiplied by URS $/ \omega^{3}$.

artifacts due to unrealistic degeneracies of the vibrations. For example, the spheroidal and torsional modes with $\ell=2$ for an isotropic system split into the first $\left(\mathrm{A}_{1 g}, \mathrm{~B}_{1 g}, \mathrm{~B}_{2 g}, \mathrm{E}_{g}\right)$ and $\left(\mathrm{A}_{1 u}, \mathrm{~B}_{1 u}, \mathrm{~B}_{2 u}, \mathrm{E}_{u}\right)$ modes respectively. The frequency splitting due to the tetragonal symmetry is quite significant. Tab. I shows that due to the U2S factor, some modes will scatter neutrons significantly more efficiently than some others. The U2S values for the torsional modes are roughly two times larger than that for the spheroidal modes making INS more sensitive to torsional modes. Because of the $1 / \omega^{3}$ factor in Eq. 11, the lowest frequency modes contribute more to $S(\omega)$. This is clearly evidenced by the variation of 
the heights of the vertical lines in Fig. 3. Therefore, the main feature in the incoherent neutron scattering function comes mainly from the scattering by the lowest $\left(\mathrm{A}_{1 u}, \mathrm{~B}_{1 u}, \mathrm{~B}_{2 u}\right.$, $\mathrm{E}_{u}$ ) modes (resulting from the splitting of the isotropic torsional mode with $\ell=2$ ).

Being based on a continuum description, the RUS model is applicable only in a limited frequency range. Optical phonons of anatase $\mathrm{TiO}_{2}$ exist down to approximately $17.8 \mathrm{meV}$ so this model cannot be trusted above this value. More importantly, the summation in Eq. 11 was performed for a limited range of $\lambda$ (the 500 lowest frequencies) which results in an even smaller frequency range where the summation converged. For sample HT5 and the size distributions used in Fig. 3, the convergence was reached for $\omega<12 \mathrm{meV}$.

\section{B. Explanations for the discrepancy between experiment and theory}

In the following, we will discuss several limitations in the model proposed in this work which could explain the discrepancy between the experimental results and the calculations. Regarding the vibrations, the applicability of continuum models is questionable especially for the modes at high frequencies or for very small sizes. However, based on comparison with existing atomistic calculations $\underline{30}, 31,32,33$, continuum approaches are valid for the sizes considered in this work at least up to the first breathing mode. In the present case, the frequency for this mode is more than 2.5 times the lowest eigenfrequency. Therefore it is reasonable to expect the present description of the vibrations to be sufficient to reproduce the main peak of the INS spectrum.

Another source of discrepancy could be the shape and crystalline structure of the NPs. In the proposed model, the NPs are perfectly spherical single domain nanocrystals. HRTEM photos $\frac{19}{19}$ confirm that this is at least a reasonable approximation. However the variations of shape or crystallinity required to shift position of the first calculated peak to the one of the experimental spectra is too large for this to be the most important reason. The same HRTEM photos also rule out the presence of a significant amount of amorphous $\mathrm{TiO}_{2}$ which could have dominated the spectrum.

Another reason for the discrepancy could be the assumption that the surface density of hydrogen atoms does not depend on the NP size. In order to better fit the calculated $S(\omega)$ to the experimental spectra, the surface density would have to increase with decreasing size

to shift the calculated first peak towards higher energies. Such a variation would be in 
agreement with a higher density of $\mathrm{OH}$ groups close to surface discontinuities. The surface of larger NPs should contain less discontinuities provided they can form larger more stable flat faces. In this case however, the variation of $d_{H}$ with $R$ would have to be very large to obtain a better agreement.

\section{Common features of INS from nanopowders and glasses}

The presently reported data for $\mathrm{TiO}_{2}$ NP powders share obvious similarities with the Boson Peak (BP) modes observed from glasses: in the acoustic regime, the INS curves evidence low frequency excitations that significantly contrast with the monotonic behaviour of the Debye density of states from extended crystalline solids. Furthermore, as in glasses, these harmonic excitations coexist with a quasielastic signal that strongly affects the visibility of the inelastic one at high temperature (Fig. 2). As in many porous systems like carbon nanotubes, zeolites or silica gels, the quasielastic signal observed in the INS spectra of $\mathrm{TiO}_{2}$ NP powders most likely arises from relaxational motions of the hydrogen atoms associated with residual adsorbed water or $\mathrm{TiOH}$ bonds.

As shown above, the low frequency excitations observed in the case of a disordered assembly of NPs essentially identify with their lowest frequency fundamental modes. Qualitatively, the inelastic bump in Fig. 1 reflects the low frequency cutoff in the vibrational density of states, below which no vibration mode can be accommodated inside the NP (see frequency gap in Fig. 3). Interestingly, such a finite size cutoff has been proposed to explain the BP origin, regardless of its spectral details that vary according the nature of the glass. $\frac{34}{4}$ The basic idea behind this interpretation is that the BP excitations are similar to NP-like modes of nanometric inhomogeneities (nanodomains) that are natively formed within the glass. The main difference between a NP assembly and a glassy nanostructure is that in the latter case the low frequency excitations are expected to be less well defined due to poor elastic contrast with the surrounding disorder (leading to hybridization with delocalized acoustic modes ${ }^{35}$ ) and also expected poor nanodomain shape definition. Nevertheless, as shown from numerical simulations, $\stackrel{36}{\underline{n}}$ the elastic disruption of the glass network at the nanometer scale suffices to generate low frequency excitations that the longstanding "continuous random network" glass model structure is unable to account for. The present INS study provides further indication that the glass BP is intrinsically related to a specific nano-texture, in line 
with previous INS studies of zeolite amorphization. $\underline{37}^{-1}$

\section{CONCLUSION}

The experimental and theoretical results presented in this paper demonstrate the possibility of using INS to observe acoustic modes confined in NPs. The experimental peaks we observed for one sample are in the right frequency range. The absence of a clear peak for the other sample with larger nanoparticles is compatible with a peak shifted towards lower frequencies as expected from our model and mostly masked by the quasielastic signal. These measurements were facilitated by the presence of hydrogen atoms due to $\mathrm{OH}$ groups at the surface. Because of the large incoherent scattering cross-section of these atoms and their increasing number with respect to the total number of atoms for decreasing NP sizes, their contribution to the INS spectra dominates for very small sizes. For larger sizes, a correct treatment would require taking into account the scattering by volume atoms which is beyond the scope of this paper. Unlike light scattering measurements, the lack of selection rules for INS enables the observation of all the vibration modes. Therefore INS measurements on samples with narrower size distributions would provide valuable experimental data to compare to current models of the vibrations of NPs.

* Electronic address: lucien.saviot@u-bourgogne.fr

† Electronic address: daniel.murray@ubc.ca

1 A. Mlayah and J. Groenen, Light scattering in solids IX (Springer-Verlag Berlin, Heidelberger Platz 3, D-14197 Berlin, Germany, 2007), vol. 108 of Topics in applied physics, section 4, pp. 237-315, 1st ed.

2 E. Duval, A. Boukenter, and B. Champagnon, Phys. Rev. Lett. 56, 2052 (1986).

3 J. Burgin, P. Langot, A. Arbouet, J. Margueritat, J. Gonzalo, C. N. Afonso, F. Vallée, A. Mlayah, M. D. Rossell, and G. V. Tendeloo, Nano Lett. 8, 1296 (2008), and references therein.

4 D. B. Murray, C. H. Netting, L. Saviot, C. Pighini, N. Millot, D. Aymes, and H.-L. Liu, J. Nanoelectron. Optoelectron. 1, 92 (2006). 
5 T.-M. Liu, J.-Y. Lu, H.-P. Chen, C.-C. Kuo, M.-J. Yang, C.-W. Lai, P.-T. Chou, M.-H. Chang, H.-L. Liu, Y.-T. Li, et al., Appl. Phys. Lett. 92, 093122 (2008).

6 B. Fultz, J. L. Robertson, T. A. Stephens, L. J. Nagel, and S. Spooner, J. Appl. Phys. 79, 8318 (1996).

7 U. Stuhr, H. Wipf, K. H. Andersen, and H. Hahn, Phys. Rev. Lett. 81, 1449 (1998).

8 E. Bonetti, L. Pasquini, E. Sampaolesi, A. Deriu, and G. Cicognani, J. Appl. Phys. 88, 4571 (2000).

9 P. M. Derlet, R. Meyer, L. J. Lewis, U. Stuhr, and H. V. Swygenhoven, Phys. Rev. Lett. 87, 205501 (2001).

10 L. Pasquini, A. Barla, A. I. Chumakov, O. Leupold, R. Rüffer, A. Deriu, and E. Bonetti, Phys. Rev. B 66, 073410 (2002).

11 A. F. Yue, A. B. Papandrew, O. Delaire, B. Fultz, Z. Chowdhuri, R. M. Dimeo, and D. A. Neumann, Phys. Rev. Lett. 93, 205501 (2004).

12 S. Rols, Z. Benes, E. Anglaret, J. L. Sauvajol, P. Papanek, J. E. Fischer, G. Coddens, H. Schober, and A. J. Dianoux, Phys. Rev. Lett. 85, 5222 (2000).

13 C. Pighini, D. Aymes, N. Millot, and L. Saviot, J. Nanoparticle Research 9, 309 (2007).

14 M. Ivanda, S. Musić, M. Gotić, A. Turković, A. M. Tonejc, and O. Gamulin, Journal of Molecular Structure 480-481, 641 (1999).

15 S. Musić, M. Gotić, M. Ivanda, S. Popović, A. Turković, A. Sekulić, and K. Furić, Materials Science and Engineering B 47, 33 (1997).

16 M. Montagna, E. Moser, F. Visintain, M. Ferrari, L. Zampedri, A. Martucci, M. Guglielmi, and M. Ivanda, Journal of Sol-Gel Science and Technology 26, 214 (2003).

17 W. M. Visscher, A. Migliori, T. M. Bell, and R. A. Reinert, J. Acoust. Soc. Am. 90, 2154 (1991).

18 H. Lamb, Proc. London Math. Soc. 13, 189 (1882).

19 C. Pighini, Ph.D. thesis, Université de Bourgogne (2006), http://tel.archives-ouvertes.fr/tel00129329 .

20 G. Li, L. Li, J. Boerio-Goates, and B. F. Woodfield, J. Am. Chem. Soc. 127, 8659 (2005).

21 R. Mueller, H. K. Kammler, K. Wegner, and S. E. Pratsinis, Langmuir 19, 160 (2003).

22 A. Y. Nosaka, T. Fujiwara, H. Yagi, H. Akutsu, and Y. Nosaka, J. Phys. Chem. B 108, 9121 (2004). 
23 T. Bezrodna, G. Puchkovska, V. Shymanovska, J. Baran, and H. Ratajczak, J. of Mol. Struct. 700, 175 (2004).

24 A. A. Levchenko, A. I. Kolesnikov, N. L. Ross, J. Boerio-Goates, B. F. Woodfield, G. Li, and A. Navrotsky, J. Phys. Chem. A 111, 12584 (2007).

25 B. Hudson, A. Warshel, and R. G. Gordon, J. Chem. Phys. 61, 2929 (1974).

26 A. C. Zemach and R. J. Glauber, Phys. Rev. 101, 118 (1956).

27 L. Saviot and D. B. Murray, Phys. Rev. B 72, 205433 (2005).

28 M. Iuga, G. Steinle-Neumann, and J. Meinhardt, Eur. Phys. J. B 58, 127 (2007).

29 H. Portalès, N. Goubet, L. Saviot, S. Adichtchev, D. B. Murray, A. Mermet, E. Duval, and M.-P. Piléni, Proc. Natl. Acad. Sci. U.S.A 105, 14784 (2008).

30 W. Cheng, S.-F. Ren, and P. Y. Yu, Phys. Rev. B 71, 174305 (2005).

31 W. Cheng, S.-F. Ren, and P. Y. Yu, Phys. Rev. B 72, 059901(E) (2005).

32 N. Combe, J. R. Huntzinger, and A. Mlayah, Phys. Rev. B 76, 205425 (2007).

33 F. Ramirez, P. R. Heyliger, A. K. Rappé, and R. G. Leisure, J. Acoust. Soc. Am. 123, 709 (2008).

34 E. Duval, A. Boukenter, and T. Achibat, J. Phys.: Condens. Matter 2, 10227 (1990).

35 E. Duval, A. Mermet, and L. Saviot, Phys. Rev. B 75, 024201 (2007).

36 F. Léonforte, R. Boissière, A. Tanguy, J. P. Wittmer, and J. L. Barrat, Phys. Rev. B 72, 224206 (2005).

37 G. N. Greaves, F. Meneau, O. Majérus, D. G. Jones, and J. Taylor, Science 308, 1299 (2005). 